The treatment of the case was.symptomatic, with rather vigorous stimulation in a child so young; she received for days, every three or four hours, hypodermics of digitalin, gr. $1 / 50$ to $1 / 100$, and nitroglycerin, gr. $1 / 200$ to $1 / 400$, together with strychnia and whisky at intervals.

The most common complications of pertussis is bronchopneumonia, and the mortality is large, more than 25 per cent. Other and less frequent complications are meningitis, measles, diphtheria, convulsions, cerebral hemorrhage and spasm of the glottis. The text-books mostly mention nephritis in the list of complications, and yet, with a rather extended search of the English literature, I was unable to find a single reported case.

The special points of interest in this case are the number and severity of the complications, any one of which would give a bad prognosis, but to have present in a child of this age, acute nephritis, with suppression of urine for about twelve hours, a rather severe type of bronchopneumonia, a complete hemiplegia due to cerebral hemorrhage, and laryngeal stenosis of rather severe type, with recovery, was more than those in attendance or consultation could have hoped for.

\section{A CASE OF VACCINATION OPHTHALMIA.}

\section{E. F. SNYDACKER, M.D. CHICAGO.}

I think the following case which came under my observation worthy of publication, as it brings before the practitioner a serious complication of vaccination but little thought of, and too little guarded against:

Willie S., $61 \frac{1}{2}$ years old, had been vaccinated about three weeks before I first saw him. The vaccination had taken, the arm had become sore and swollen, there was much discharge from the point of vaccination, also much itching. Although the point of vaccination had been protected by a shield, the child had raised this and scratched the wound. About two weeks after vaccination the eye became red, and the lids became swollen, till the eye was almost completely closed. When the mother brought the child to me the condition was as follows:

The lids were swollen so as to close the eye almost completely; a profuse mucopurulent secretion was present. On opening the lids, a gray membrane was seen covering the inner portion of the eyeball, and the conjunctival surface of the lids, extending from the caruncle to the cornea, and covering both upper and lower tear puncta. This membrane was moderately thick, but little adherent, and caused but little bleeding on removal. On the conjunctival surface of the lower lid, near the punctum, but not involving it, was a swollen oval ulcer with ragged, dirty gray edges; here the membrane was somewhat more adherent and there was bleeding on removing the membrane. The cornea was hazy, and at its inner border was a small gray urea of infiltration about the size of a pinhead. The pupil was contracted, but the iris was not discolored and reacted to light.

At first I thought I had to do with a case of diphtheria. I took the child's temperature and found it normal. I examined the nose and throat and found no involvement there. The mother herself called my attention to the fact that the child had been vaccinated, and that she thought there was some connection between the inflammation of the eye and the vaccina. tion. It then began to dawn on me that $I$ was dealing with a case of inoculation of the eye with vaccine virus.

I removed the membrane, and by means of small cotton swab I carefully and thoroughly touched its site, as well as the site of the conjunctival ulcer of the lid with a solution of permanganate of potash, 1 to 250 . I instilled a 1 per cent. atropin solution into the eye, and found that the pupil dilated evenly and without difficulty. The mother being unwilling to Jeave the child in the hospital, I ordered a 10 per cent. argyrol solution to be dropped into the eye four times a day, atropin solution (I grain to the ounce) twice a day, hot applications four times a day, and I myself applied a 1 to 250 solution of permanganate of potash daily to the conjunctiva of the lids. A culture from the membrane showed no Klebs-Loeffler bacillus.

At first the condition proved somewhat refractory. While the membrane did not reform, the conjunctival ulcer was slow in healing, and the corneal infiltration showed a tendency to spread, becoming thicker, grayer and larger, the epithelium breaking down, till there was an ulcer about half the size of a split pea. It was only after cauterizing the corneal ulcer twice thoroughly with a 95 per cent. solution of carbolic acid that it was checked. In all, it took four weeks of careful attention before the boy was discharged cured, and when last I saw him, six months after his discharge, there was still quite a dense gray macula at the site of the corneal ulcer, which fortunately involved only the periphery of the cornea, and did not, therefore, injure the vision in the least.

The literature does not deal very extensively with this condition. The case that lave been reported are carefully analyzed in a dissertation by Dr. Reiner Schmitz of Dortmund. He cites cases of Berry, Critchell, Hirschberg, Peiper, Schapringer, Schirmer, Sénut and Weiss. Aron also reported several cases and reviewed the literature.

While in the majority of these cases the outcome was good, at times, the eye involvement was very severe, and in several cases severe corneal complications resulted in loss of sight. If we happen to know that an individual presenting such an eye involvement has been vaccinated, and are fortunate enough to have somebody question the connection between the two conditions, then a diagnosis is easy; otherwise, we are almost certain, if we see the disease during its membranous stage, to mistake it for diphtheria.

The differential diagnosis, however, is not difficult. The fact that the membrane is not especially adherent, that it does not bleed freely on removal, the absence of nose and throat involvement and of severe constitutional symptoms, and, finally, the culture test render a differential diagnosis easily possible.

103 State Street.

\section{DEATH FOLLOWING ADMINISTRATION OF A CROUP REMEDY.}

\section{J. A. PALMER, M.D ERIE, KAN.}

I wish to report a case in which death was directly attributable to "Dr. Drake's German Cough Remedy."

I was called to attend a child, aged 4 years, who was in deep coma. Breathing was stertorous, skin cyanotic, pulse intermittent and almost imperceptible, pupils were deeply contracted, in fact, I saw at once that the child was dying of opium poisoning. On inquiry, I found that the child had had an attack of croup the previous evening and had been given one-half teaspoonful of Dr. Drake's German Croup Remedy every half hour. The child died within an hour after I saw it, and was sick, in all, about twenty-four hours.

This preparation is said to contain three-fourths of a grain of opium to the dose, and the child had been given about half the contents of the bottle-which contained about three ounces-an enormous amount of opium for a child of this age. It is stated on the label that the dose is so small as to be harmless. Were the equivalent in minims or drops of laudanum stated on the label, the public might have a better appreciation of the danger of administering nostrums containing opium. When the amount is expressed in grains it means little except to a physician. In this case the statement that the amount of opium present is so small as to be harmless is little short of criminal. 